a 6-monthly LDL-C $>1.8 \mathrm{mmol} / \mathrm{L}$ and were on high dose statin therapy, we included these patients in our final analysis, resulting in $37.1 \%(111 / 299)$ patients in our cohort being eligible for a PCSK9i based on current ESC guidelines, results are summarized in figure 1.

Discussion Our results highlight the discrepancy between current ESC/EAS guidelines and the reimbursement criteria for PCSK9i in Ireland. This discrepancy results in minimal patients with coronary artery disease being eligible for a PCSK9i in Ireland. The results also highlight that a significant proportion of patients in clinical practice do not meet LDL-C goals post cardiac rehabilitation, these targets should be met in order to reduce future cardiovascular risk and improve outcomes for patients.

\section{IMPACT OF MORBID OBESITY AND OBESITY PHENOTYPE ON OUTCOMES POST TRANSCATHETER AORTIC VALVE REPLACEMENT}

${ }^{1} \mathrm{~A}$ Mclnerney, ${ }^{1} \mathrm{G}$ Tirado-Conte, ${ }^{2} \mathrm{~J}$ Rodes-Cabau, ${ }^{3} \mathrm{~F}$ Campelo-Parada, ${ }^{4} \mathrm{JD}$ Tafur Soto, ${ }^{5} \mathrm{M}$ Barbanti, ${ }^{6} \mathrm{E}$ Muñoz-Garcia, ${ }^{7} \mathrm{M}$ Arif, ${ }^{8} \mathrm{D}$ Lopez, ${ }^{9} \mathrm{~S}$ Toggweiler, ${ }^{10} \mathrm{G}$ Veiga, ${ }^{11} \mathrm{~A}$ Pylko, ${ }^{12} \mathrm{~T}$ Sevilla, ${ }^{13} \mathrm{M}$ Compagnone, ${ }^{14} \mathrm{~A}$ Regueiro, ${ }^{15} \mathrm{~V}$ Serra, ${ }^{1} \mathrm{M}$ Carnero, ${ }^{16} \mathrm{JF}$ Oteo, ${ }^{17} \mathrm{~F}$ Rivero, ${ }^{18} \mathrm{H}$ Barbosa Ribeiro, ${ }^{2} \mathrm{~L}$ Guimaraes, ${ }^{3} \mathrm{~A}$ Matta, ${ }^{4} \mathrm{~N}$ Giraldo Echavarria, ${ }^{5} \mathrm{R}$ Valvo, ${ }^{9} \mathrm{~F}$ Moccetti, ${ }^{6} \mathrm{AJ}$ Muñoz-Garcia, ${ }^{8} \mathrm{~J}$ Lopez-Pais, ${ }^{15} \mathrm{~B}$ Garcia del Blanco, ${ }^{18} \mathrm{D}$ Carter Campanha Borges, ${ }^{1} \mathrm{~N}$ Gonzalo, ${ }^{2} \mathrm{E}$ Dumont, ${ }^{5} \mathrm{E}$ Criscione, ${ }^{11} \mathrm{M}$ Dabrowski, ${ }^{17} \mathrm{~F}$ Alfonso, ${ }^{10} \mathrm{JM}$ de la Torre Hernández, ${ }^{7} \mathrm{AN}$ Cheema, ${ }^{12} \mid$ Amat-Santos, ${ }^{13} \mathrm{~F}$ Saia, ${ }^{1} \mathrm{~J}$ Escaned, ${ }^{1} \mathrm{~L}$ Nombela-Franco. ${ }^{1}$ Cardiovascular Institute. Hospital Clínico San Carlos. IdISSC. Madrid. Spain; ${ }^{2}$ Quebec Heart and Lung Institute, Laval University, Quebec City, Quebec, Canada; ${ }^{3}$ Cardiology Department, Rangueil University Hospital, Toulouse, France; ${ }^{4}$ The Ochsner Clinical School, Ochsner Medical Center, New Orleans, LA, US; ${ }^{5}$ Ferrarotto Hospital, University of Catania, Catania, Italy; ${ }^{6}$ CIBERCV Cardiology Department, Hospital Universitario Virgen de la Victoria, Málaga, Spain; 'Division of Cardiology, St. Michael's Hospital, Toronto University, Toronto, Ontario, Canada; ${ }^{8}$ Hospital Clínico Universitario de Santiago, CIBERCV, Santiago, Spain; ${ }^{9}$ Heart Center Lucerne, Luzerner Kantonsspital, Lucerne, Switzerland; ${ }^{10}$ Hospital Universitario Marques de Valdecilla, Santander, Spain; ${ }^{11}$ Interventional Cardiology and Angiology Clinic, Institute of Cardiology, Warsaw, Poland; ${ }^{12}$ CIBERCV, Instituto de Ciencias del Corazón (ICICOR), Hospital Clínico Universitario de Valladolid, Valladolid, Spain; ${ }^{13}$ Cardiology Unit, Cardio-Thoracic-Vascular Department, University Hospital of Bologna, Policlinico S. Orsola - Malpighi, Bologna, Italy; ${ }^{14}$ Cardiology Department, Cardiovascular Institute, Hospital Clínic; Universidad de Barcelona; Institut d'Investigacions Biomèdiques August Pi i Sunyer, Barcelona, Spain; ${ }^{15}$ Hospital General Universitari Vall d'Hebron, Barcelona, Spain; ${ }^{16}$ Department of Cardiology, Hospital Universitario Puerta de Hierro, Majadahonda, Spain; ${ }^{17}$ Department of Cardiology, Hospital Universitario La Princesa, Madrid, Spain; ${ }^{18}$ Heart Institute (InCor), Sao Paulo, Brazil

\subsection{6/heartjnl-2020-ICS.3}

Background The obesity epidemic continues to grow and coupled with an ageing population the number of obese patients undergoing transcatheter aortic valve replacement (TAVR) has increased significantly. Morbidly obese (MO) patients however, are underrepresented in trials, and there is conflicting evidence regarding their outcomes post TAVR.

Aim The aim of our study was to compare periprocedural and mid-term outcomes in a matched cohort of $\mathrm{MO}$ and nonobese (NO) patients and to determine whether adipose tissue distribution analysis from pre TAVR CT scans can provide prognostic information.

Methods This was a multicentre study involving 18 tertiary referral centres in Europe, Canada, North and South America. Consecutive MO patients (BMI $\geq 40 \mathrm{~kg} / \mathrm{m}^{2}$, or $\geq 35 \mathrm{~kg} / \mathrm{m}^{2}$ with obesity related co-morbidities) with severe aortic stenosis (AS) who underwent TAVR were analyzed and compared to a non-obese (NO) cohort (BMI 18.5-29.9 $\mathrm{kg} / \mathrm{m}^{2}$ ). Data on patient characteristics, periprocedural and mid-term outcomes were collected.
A propensity-score matched analysis was performed matching NO and MO patients on a 1:1 basis to assess differences in outcomes between groups. A multivariate analysis was undertaken to determine predictive factors for all-cause mortality at 2 years in the MO group.

Pre-TAVR computed tomography scans were analyzed in a centralized core laboratory to assess adipose tissue distribution based on epicardial fat (EAT), abdominal visceral (VAT) and subcutaneous fat (SAT), and to evaluate its impact on outcomes.

Results A total of 3174 patients undergoing TAVR were included: 2264 in the NO and 910 in the MO groups respectively. After propensity score application, a matched cohort with 770 patients per group was obtained. Groups were well matched although some baseline cardiovascular risk factors, such as hypertension, hyperlipidemia and insulin requiring diabetes mellitus, continued to differ. Major vascular complications (MVC) occurred more commonly $(6.6 \%$ vs $4.3 \%$, $\mathrm{p}=0.043)$ and device success was lower $(84.4 \%$ vs $88.1 \%$, $\mathrm{p}=0.038$ ) in the $\mathrm{MO}$ group. After a median follow up of 14.11 months [IQR 6.47-36.01], survival analysis demonstrated similar rates of all-cause and cardiovascular mortality for matched $\mathrm{MO}$ and $\mathrm{NO}$ groups (79.4 vs $80.6 \% \mathrm{p}=0.731$ and 88.7 vs $87.4 \% \mathrm{p}=0.699$ respectively). Decreased baseline hemoglobin, non-transfemoral vascular access, MVC, stage 2-3 acute kidney, and periprocedural stroke were all independent predictors of 2-year mortality.

Adipose tissue distribution analysis identified an adverse MO phenotype whereby abdominal VAT:SAT ratio $\geq 1$ was associated with increased 2-year all-cause (HR 3.06, 95\%CI 1.20-7.77, $\mathrm{p}=0.019$ ) and cardiovascular (HR 4.11, 95\%CI $1.06-15.90, \mathrm{p}=0.041$ ) mortality, and readmissions (HR 1.81, 95\%CI 1.07-3.07, $\mathrm{p}=0.027)$. Additionally, increased all-cause mortality at 2 years was found for each $10 \mathrm{~cm}^{3} / \mathrm{m}^{2}$ increment in indexed EAT (HR 1.16, 95\% CI 1.03-1.30, $\mathrm{p}=0.011$ ). After multivariable analysis, VAT:SAT ratio $\geq 1$ remained a strong predictor of 2-year mortality (HR 2.78, $\mathrm{p}=0.035$ ).

Conclusion MO patients undergoing TAVR demonstrate similar periprocedural and mid-term outcomes to a matched cohort of NO patients. Higher rates of MVC in the MO group highlights the need for vigilance when performing vascular access. VAT:SAT $\geq 1$ identifies an obesity phenotype at higher risk of 2 -year mortality and readmission.

\section{PREDICTORS OF VENTRICULAR ARRHYTHMIA IDENTIFIED FROM FOLLOW UP OF TETRALOGY OF FALLOT}

${ }^{1} \mathrm{~B}$ Kelly, ${ }^{1} \mathrm{PF}$ Brennan, ${ }^{1} \mathrm{C}$ Lockhart, ${ }^{1} \mathrm{M}$ Spence, ${ }^{1} \mathrm{C}$ Owens, ${ }^{2} \mathrm{~A}$ Sands, ${ }^{2} \mathrm{~B}$ McCrossan, 1J Gordon, 'L Briggs. 'Adult Congenital Heart Disease department, Royal Victoria Hospital, Belfast, UK; ${ }^{2}$ Paediatric Cardiology Department, Royal Belfast Hospital Sick Children, Belfast, UK

\subsection{6/heartjn|-2020-ICS.4}

Introduction Tetralogy of Fallot (TOF) is the most common cyanotic congenital heart defect. Subsequent to the introduction of surgical repair, long-term outcomes for patients with TOF have improved significantly. However right ventricular outflow tract dysfunction and regurgitative volume overload remains a recognised sequalae, often progressing to right ventricular (RV) dilatation and dysfunction, arrhythmia, and premature death. In repaired TOF ( $\mathrm{rToF})$, QRS prolongation is a recognised predictor of the development of sustained 
ventricular tachycardia (VT) and sudden cardiac death. Ventricular dysfunction, atrial tachyarrhythmias and RV hypertrophy are also predictive of sustained VT and death in rToF. We, retrospectively, report the overall long-term survival of rToF from our single tertiary centre.

Methodology For all 229 consecutive patients with TOF, baseline clinical characteristics, echocardiography and magnetic resonance imaging results, documented evidence of arrhythmia and analysis of outcomes were collected retrospectively using Northern Ireland Electronic Care Record (NIECR) and our dedicated in-hospital databases. Causes of death were elicited using NIECR +/or the patients primary care physician. We analysed all-cause mortality as well as cardiovascular mortality. Cardiovascular mortality was subsequently divided into sudden cardiac/arrhythmic death and expected cardiac death. Additionally, we analysed the incidence of confirmed ventricular arrhythmia defined as 33 consecutive ventricular complexes. Survival was estimated using Kaplan-Meier analysis. Univariate predictors of sudden cardiac death (SCD) or the occurrence of ventricular events (VE) during follow-up were assessed via binary logistic regression and a Cox proportional hazards model. Clinically predictors on univariate analysis, based on an $\alpha$-level $<0.01$, were subsequently imputed into a multivariate model.

Results The mean patient age was $34 \pm 11$ years (range 1668 years $)$ and $57 \%(n=131)$ are male. $6.1 \%(n=14)$ patients have died from all-causes during follow-up. Of the 14 deaths, SCD occurred in 50\% $(\mathrm{n}=7)$ patients, expected cardiac death in $21 \%(n=3)$ patients, and non-cardiovascular causes accounted for the remaining 29\% $(n=4)$ patients. The median survival estimate was 65 years age (95\% C.I 61.0268.98). ECG-documented or device captured ventricular events occurred in $11 \%(n=26)$ patients during follow-up. On univariate analysis, the presence of late gadolinium enhancement (LGE) scar on CMR $(p<0.005$, OR 18.20), LVEF $<50 \%(p<0.005$, OR 7.21), QRS $>160 \mathrm{~ms}$ (p 0.01, OR 3.12) and AF (p 0.02, OR 4.66) were all associated with increased odds of VE. In the multivariate model both impaired LV systolic function (LVEF<50\%, p 0.04, HR 5.63) and late gadolinium enhancement (LGE) scar on CMR ( $p$ $<0.005$, HR 11.42) were significant predictors of VE during follow-up, as summarised in table 1.

Conclusion Amongst patients with rToF, presence of LGE scar on CMR, QRS $>160 \mathrm{~ms}, \mathrm{LVEF}<50 \%$ and $\mathrm{AF}$ are associated with increased occurrence of ventricular events. These findings represent a contemporary cohort of ToF patients and whilst

Abstract 4 Table 1 Predictors of captured ventricular events (VE)

\begin{tabular}{llllll}
\hline \multicolumn{3}{c}{ Univariate analysis } & \multicolumn{5}{c}{ Multivariate analysis } \\
\hline Variable & OR & p value & HR & P value & $95 \%$ C.I \\
\hline QRS $>160 \mathrm{~ms}$ & 3.12 & 0.01 & 3.66 & 0.06 & $0.96-14.02$ \\
RVEDVi $>160 \mathrm{ml} / \mathrm{m}^{2}$ & 0.22 & 1.85 & & & \\
RVESVi $>100 \mathrm{ml} / \mathrm{m}^{2}$ & 1.76 & 0.34 & & & \\
RVEF $<50 \%$ & 0.70 & 0.52 & & & \\
LVEF $<50 \%$ & 7.21 & 0.001 & 5.63 & 0.04 & $1.09-29.10$ \\
NTpro-BNP $>400 \mathrm{pg} / \mathrm{L}$ & 2.43 & 0.11 & & & \\
AF & 4.66 & 0.02 & & & \\
Pulmonary Valve & 1.65 & 0.23 & & & \\
replacement/repair & & & & & \\
LGE scar on CMR & 18.20 & $<0.005$ & 11.42 & $<0.005$ & $2.97-43.90$ \\
\hline
\end{tabular}

have been described previously, add weight to the potential for risk scoring algorithms in ToF to prevent sudden death.

\section{Oral abstract presentations 1}

\section{CLINICAL PREDICTION SCORES WITH OR WITHOUT NTPROBNP IN PREDICTING STROKE AND TRANSIENT ISCHEMIC ATTACK IN AT-RISK PATIENTS WITHOUT ATRIAL FIBRILLATION - THE STOP-HF EXPERIENCE} ${ }^{1,2} \mathrm{~B}$ Kerr, ${ }^{1} \mathrm{~L}$ Brandon, ${ }^{1} \mathrm{~V}$ O'Sullivan, ${ }^{1,2} \mathrm{R}$ Pharithi, ${ }^{1} \mathrm{C}$ Sweeney, ${ }^{1} \mathrm{~F}$ Ryan, ${ }^{2} \mathrm{~J}$ Gallagher,
${ }^{1,2} \mathrm{~K}$ McDonald, ${ }^{1,2} \mathrm{M}$ Ledwidge. ' STOP-HF Unit, St. Vincent's University Hospital Healthcare
Group, Elm Park, Dublin 4, Ireland; ${ }^{2}$ University College Dublin, Ireland

\subsection{6/heartjnl-2020-ICS.5}

Background Clinical prediction rules, such as CHA2DS2-VASc, $\mathrm{HATCH}$ and HAVOC have been used in the prediction of stroke and transient ischemic attack (TIA) in those with atrial fibrillation (AF). Currently the ESC guidelines recommend the use of the CHA2DS2-VASc score to help determine which patients with AF would most benefit from anticoagulation. In recent years there has been a trend to use CHA2DS2-VASc for stroke prediction even in the non-AF population, with similar C-statistic (0.61-0.76) in comparison to AF cohorts. Similarly, natriuretic peptides have gained interest as a marker of cardiovascular risk, including cardioembolic stroke.

Purpose We compared CHA2DS2-VASc, HA2TCH and HAVOC for prediction of stroke/TIA in patients without a history of atrial fibrillation or flutter and hypothesised that

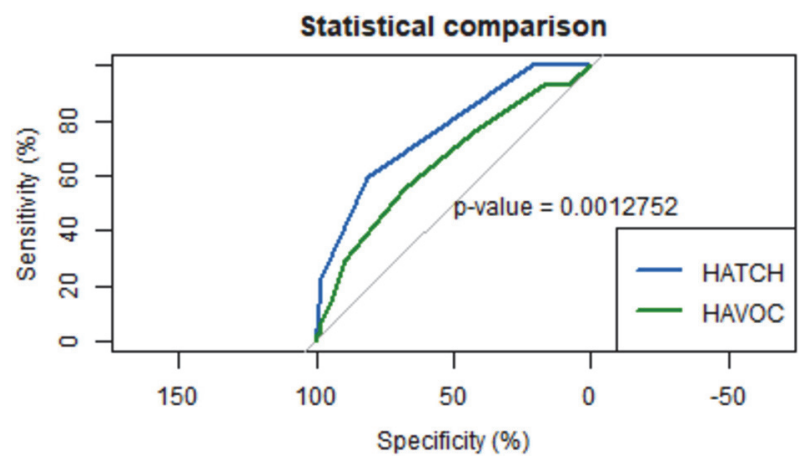

Abstract 5 Figure 1 Comparing Receiver operating curves(ROC) of the HATCH and HAVOC scores

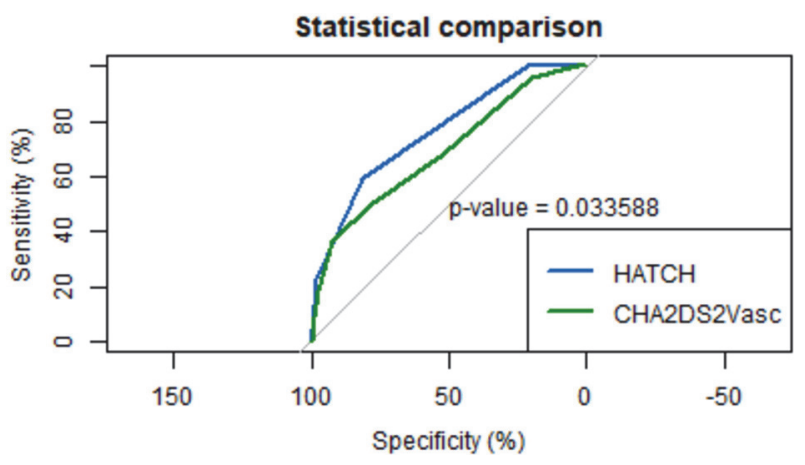

Abstract 5 Figure 2 Comparing Receiver operating curves(ROC) of the HATCH and $\mathrm{CHA}_{2} \mathrm{DS}_{2}$-VASc scores 